\title{
Large-Scale Expansion of the Face Representation in Somatosensory Areas of the Lateral Sulcus after Spinal Cord Injuries in Monkeys
}

\author{
Shashank Tandon, Niranjan Kambi, Leslee Lazar, Hisham Mohammed, and Neeraj Jain \\ National Brain Research Centre, Manesar 122050, India
}

\begin{abstract}
Transection of dorsal columns of the spinal cord in adult monkeys results in large-scale expansion of the face inputs into the deafferented hand region in the primary somatosensory cortex (area $3 \mathrm{~b}$ ) and the ventroposterior nucleus of thalamus. Here, we determined whether the upstream cortical areas, secondary somatosensory (S2) and parietal ventral (PV) areas, also undergo reorganization after lesions of the dorsal columns. Areas S2, PV, and 3b were mapped after long-term unilateral lesions of the dorsal columns at cervical levels in adult macaque monkeys. In areas S2 and PV, we found neurons responding to touch on the face in regions in which responses to touch on the hand and other body parts are normally seen. In the reorganized parts of S2 and PV, inputs from the chin as well as other parts of the face were observed, whereas in area $3 \mathrm{~b}$ only the chin inputs expand into the deafferented regions. The results show that deafferentations lead to a more widespread brain reorganization than previously known. The data also show that reorganization in areas S2 and PV shares a common substrate with area 3b, but there are specific features that emerge in S2 and PV.
\end{abstract}

\section{Introduction}

Brains of adult mammals remain remarkably plastic. The primary somatosensory cortex undergoes somatotopic reorganization after injuries to the peripheral or spinal pathways (Jones, 2000; Chen et al., 2002; Jain, 2002; Kaas et al., 2008). Deafferentations that deprive small regions of the cortex of its normal inputs, such as transection of the nerves to the hand or amputation of one or more digits, lead to expansion of remaining inputs from the hand into the deprived cortex (Merzenich et al., 1983a,b, 1984; Garraghty and Kaas, 1991a). Such reorganization, which is confined within the representation of the same body part, has also been reported from a variety of other mammalian species (Wall and Cusick, 1984; Calford and Tweedale, 1988; Turnbull and Rasmusson, 1991; Dykes et al., 1995; McCandlish et al., 1996). Injuries that deprive a large expanse of the cortex, such as lesions of the dorsal columns of the spinal cord (Jain et al., $1997,2000,2008$ ) or transection of the spinal dorsal roots (Pons et al., 1991) lead to what has been termed "large-scale" reorganization. Such injuries result in expansion of inputs from the face into the deprived hand cortex. The face expansion in area $3 \mathrm{~b}$ is generally reported to be $\sim 10-12 \mathrm{~mm}$ (Pons et al., 1991; Jain et al., 2000 ), although expansion by as much as $20 \mathrm{~mm}$ was seen in one monkey (Jain et al., 2008). A more limited medial expansion of

Received April 30, 2009; revised Aug. 11, 2009; accepted Aug. 16, 2009.

This work was supported by Wellcome Trust International Senior Research Fellowship (N.J.); N.J. also acknowledges full salary support from the Trust Grant 063259/Z/00/Z. Financial support from the core funds of National Brain Research Centre is gratefully acknowledged. We thank Dr. V. Rema for helpful comments on this manuscript, Radhika Rajan for her help during mapping experiments, and Mithlesh Singh and Harishankar for technical help.

Correspondence should be addressed to Neeraj Jain, National Brain Research Centre, N.H. 8, Manesar 122050, India. E-mail: neeraj.jain@nbrc.ac.in or njain.nbrc@gmail.com.

DOI:10.1523/JNEUROSCI.2118-09.2009

Copyright $\odot 2009$ Society for Neuroscience 0270-6474/09/2912009-11\$15.00/0 the face inputs is also seen after loss of a limb in humans and nonhuman primates (Flor et al., 1995; Florence and Kaas, 1995).

Previous studies on reorganization of the somatosensory cortex after injuries to the peripheral or spinal pathways have generally focused on area $3 \mathrm{~b}$. In addition, reorganization in area 1 (Merzenich et al., 1983a; Garraghty et al., 1990a), the ventroposterior (VP) nucleus of thalamus (Garraghty and Kaas, 1991b; Lenz et al., 1994; Florence and Kaas, 1995; Davis et al., 1998; Jain et al., 2008), and the cuneate nucleus of brainstem (Xu and Wall, 1997, 1999; Darian-Smith and Ciferri, 2006) have also been reported. However, it is not known whether upstream cortical areas, the second somatosensory area (S2) and the parietal ventral area $(\mathrm{PV})$, which retain direct access to intact spinothalamic inputs after dorsal column lesions, also undergo reorganization.

It has been proposed that somatotopic reorganization results in perceptual abnormalities such as phantom sensations, which are experienced by patients with spinal cord injuries or amputations (Ramachandran et al., 1992; Flor et al., 1995; Kaas et al., 1999; Jain, 2002). This proposal implies that topographically inappropriate sensory inputs must reach higher areas of the brain, and merge into the preinjury, intact percept of the body, the body schema, or neuromatrix (Head and Holmes, 1912; Bromage and Melzack, 1974). Thus, the postinjury state of areas S2 and PV could provide an important substrate for phantom sensations.

We made unilateral lesions of the dorsal columns at cervical levels in adult macaque monkeys and determined whether areas $\mathrm{S} 2$ and PV undergo topographic reorganization. Areas S2 and PV were mapped after $>1$ year of recovery period. Area $3 \mathrm{~b}$ was also mapped in the same monkeys for comparison.

\section{Materials and Methods}

Animals. Five adult macaque monkeys (Macaca mulatta), four males and one female, were used for these experiments. Three monkeys, LM01, 
LM31, and LM34, received lesions of the dorsal columns of the spinal cord. The monkeys weighed $5.9,11.8$, and $8.8 \mathrm{~kg}$, respectively, at the time of the lesion. Areas S2 and PV were also mapped in two normal monkeys, NM14 and NM116, which weighed 3.7 and $3.3 \mathrm{~kg}$ at the time of mapping. Data from two other adult monkeys (NM24 and NM36) were used to determine receptive field (RF) sizes for neurons in normal area $3 \mathrm{~b}$ (see Results). All animal protocols were approved by the Animal Ethics Committee of the National Brain Research Centre, and the Committee for the Purpose of Control and Supervision of Experiments on Animals, Government of India, and conformed to National Institutes of Health guidelines.

Dorsal column lesions. Unilateral dorsal column lesions were made at cervical levels in three monkeys under sterile conditions. The monkeys were anesthetized using a mixture of ketamine $(8 \mathrm{mg} / \mathrm{kg}$, i.m.) and xylazine $(0.4 \mathrm{mg} /$ $\mathrm{kg}$, i.m.). Supplemental doses of anesthetics were given at one-tenth of the initial dose as required. The surgical site was prepared, a midline incision was made above the dorsal spinal cord, and the muscles were retracted. After laminectomy, the dura was incised and retracted, and dorsal columns were lesioned on one side at cervical levels using a pair of sharp fine forceps. The side for the lesion was selected so as to minimize damage to surface blood vessels. After the lesion, the dura was folded back in place, the spinal cord was covered with gel foam, and the muscles and the skin were sutured in layers. The heart rate, the core body temperature, and blood oxygenation levels were continuously monitored during the entire procedure. Animals were given antibiotics, analgesics, and dexamethasone (in reducing dose) for $5 \mathrm{~d}$ after surgery. During the recovery period, the animals were carefully monitored for food and fluid intake, or any signs of selfinjury. No complications were observed and recoveries were uneventful. For additional details of the surgical procedures, see Jain et al. (1997, 2008).

Mapping of the somatosensory cortex. We mapped area $3 \mathrm{~b}$ and areas S2 and PV in three monkeys $>1$ year after dorsal column lesions (see Fig. 1). In addition, two monkeys were mapped to determine the normal organization of areas S2 and PV. For mapping, animals were anesthetized with ketamine $(8 \mathrm{mg} / \mathrm{kg}$, i.m.) and xylazine $(0.4 \mathrm{mg} / \mathrm{kg}$, i.m.). Anesthesia was maintained by additional doses of ketamine and xylazine as required and supplemented with urethane $(250 \mathrm{mg} / \mathrm{kg}$, i.p. $)$. Dexamethasone $(2 \mathrm{mg} / \mathrm{kg}$, i.m.) was administered to prevent swelling of the brain. The heart rate and blood oxygenation levels were continuously monitored during the entire procedure. The core body temperature was monitored with a rectal probe and maintained at $37^{\circ} \mathrm{C}$ with a warmwater blanket placed under the monkey. Saline was administered intravenously, alternating with dextrose (5\% in saline) every $4 \mathrm{~h}$. The monkeys were given enrofloxacin $(5 \mathrm{mg} / \mathrm{kg}$, i.m.) and glycopyrrolate $(0.015 \mathrm{mg} / \mathrm{kg}$, i.m.) every $24 \mathrm{~h}$ during the long mapping sessions.

Tungsten microelectrodes ( $1 \mathrm{M} \Omega$ at $1 \mathrm{kHz}$; Microprobe) were used to record from the somatosensory areas. For recordings from area $3 \mathrm{~b}$, the electrode was inserted in the postcentral gyrus perpendicular to the brain surface. Penetration points were placed $\sim 500 \mu \mathrm{m}$ from each other. Receptive fields of neurons were determined at recording sites separated by

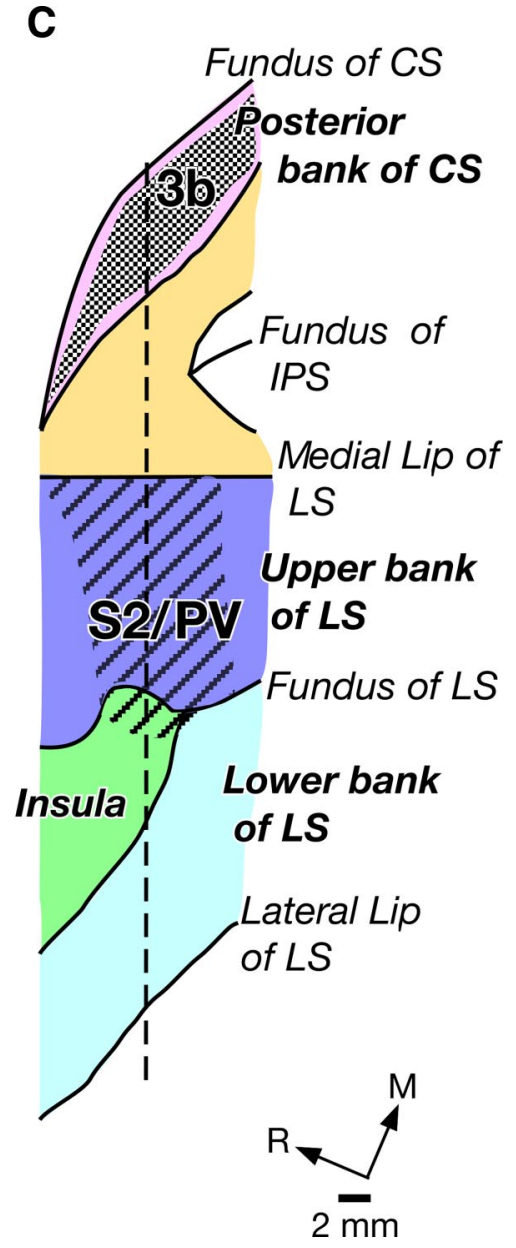

Figure 1. Location of area $S 2$ and PV in macaque monkeys. $A$, A dorsolateral view of the macaque brain showing locations of the lateral sulcus (LS), intraparietal sulcus (IPS), and central sulcus (CS). The dashed line marks the location from where the section the brain showing locations of area 3b (stippled) in the posterior bank of CS, and areas S2 and PV (hatched) in the upper and inne nk of LS. The primary auditory cortex (Aud) is labeled for reference. The colored regions between sulcal landmarks correspond to cosponding to the medial lip of $L S$ is drawn as a straight line. For details of the flattening procedure, see Materials and through CS and LS from monkey LM31. The cortex was cut at an angle approximately perpendicular to the lateral sulcus ( band the primary auditory cortex (Aud) are easily seen because of a darkly stained, prominent layer 4 . Area 4 or the primary motor cortex (M1), area 3a, and area 1 are also marked. These areas were demarcated using adjacent sections stained for Nissl substance, acetylcholine esterase, or myelin. This section was caudal to the section outlined in $\boldsymbol{B}$.

$300-400 \mu \mathrm{m}$ intervals along the depth of the sulcus. For mapping areas S2 and PV, the electrode was inserted either parallel to the upper bank of the lateral sulcus (three monkeys; two with lesion and one normal), or at an angle approximately perpendicular to the upper bank of the lateral sulcus (two monkeys; one with lesion and one normal) (Krubitzer et al., 1995). The penetrations covered the upper bank of lateral sulcus, the insular cortex, and parts of the lower bank of the lateral sulcus. The total number of penetrations made in each monkey was based on our goal to sample the organization of large portions of area $3 \mathrm{~b}$ as well as areas $\mathrm{S} 2$ and $\mathrm{PV}$, while maintaining an optimal experimental duration. The mapping sessions lasted 3-4 d for each monkey. For determining RFs of neurons, the body surface was stimulated by stroking the skin or hair with brushes, or by tapping the skin with handheld wooden probes. The body parts were also stimulated by tapping the muscles with fingers, pressing by hand, or manipulating the joints. Responses of neurons were ascertained by visual inspection of traces of action potentials on an oscilloscope and 
using an audio amplifier. The RFs were drawn on photographs of body parts of the monkeys. At all the recording sites, responses to the stimulation of all parts of the body, including the face, were determined. The experimenter mapping the RFs was unaware of the location of the electrode in the brain. Toward the end of the mapping sessions, small electrolytic lesions were made at specific sites to determine the angle of the electrode tracks and to help align the somatotopic maps and histological sections of the cortex (see Fig. 1D). The lesions were made either by passing 10 or $20 \mu \mathrm{A}$ cathodal current for $10 \mathrm{~s}$, or by continuously passing $10 \mu \mathrm{A}$ current as the electrode was withdrawn from the brain at a rate of $25 \mu \mathrm{m} / \mathrm{s}$. For other details of the mapping procedures, see Jain et al. (1997, 2008).

Histology. After completion of the recording sessions, monkeys were killed with a lethal dose of pentobarbital $(17.5 \mathrm{mg} / \mathrm{kg}$, i.v.) and perfused transcardially with $\mathrm{PBS}(0.9 \% \mathrm{NaCl}$ in phosphate buffer, $0.1 \mathrm{~m}, \mathrm{pH} 7.4)$, followed by 2 or $3 \%$ paraformaldehyde, and then by 2 or $3 \%$ paraformaldehyde in $10 \%$ sucrose. The brain and the spinal cord were removed and cryoprotected overnight in 30\% sucrose. The brain was blocked, frozen, and cut into 50 - or $60-\mu \mathrm{m}$-thick sections on a sliding microtome at an angle perpendicular to the lateral sulcus. The spinal cord was cut in a horizontal plane to reconstruct the lesion site. Different series of sections from the brain were stained for cytochrome oxidase (CO) activity (Wong-Riley, 1979), Nissl substance, acetylcholine esterase (AChE) activity (Geneser-Jensen and Blackstad, 1971), or myelin (Jain et al., 1998) to delineate boundaries between different cortical areas and to visualize the electrolytic lesions (see Fig. $1 D$ ).

Reconstruction of the spinal lesions. Sections of the spinal cord (see Fig. $4 D$ ) were used to reconstruct the spinal cord in a coronal plane. Drawings of the sections were made using a microscope equipped with camera lucida. The maximal extent of the lesion and the widths of the gray and the white matter were measured for each section and plotted on a graph paper to reconstruct the spinal cord and the extent of the lesion (Jain et al., 2008).

Reconstruction of the somatotopic maps. Sections of the cortex stained for Nissl substance were drawn using a microscope equipped with camera lucida. Location of layer 4 was marked on these drawings (see Fig. $1 B)$. Architectonic boundaries of cortical areas of the lateral sulcus and the anterior parietal cortex were marked with the help of Nissl-, AChE-, myelin-, and CO-stained sections. Locations and angles of the electrode tracks were determined with the help of the electrolytic lesions and marked on the drawings of the sections. The cortical surface was reconstructed in a flattened plane using these drawings as described below.

The drawings were scanned using a flatbed scanner and imported into Canvas X software (ACD Systems) for additional processing. The linear dimension of the cortex along layer 4 was measured and drawn as a straight line for each section (see Fig. $1 C$, dashed line). Distances between the sulcal landmarks (lips and fundi of the sulci) and architectonically defined areal boundaries were measured and marked on the line for that section. The lines depicting all the sections were aligned to each other such that the points marking the upper lip of the lateral sulcus were in a straight line (see Fig. 1C). Corresponding points for boundaries of cortical areas and sulci were joined to reconstruct a flattened view of the cortex. Electrode tracks and recording sites along the tracks were transferred to the flattened view of the cortex. The topographic maps were completed by drawing boundaries around mapping sites as per standard conventions (Jain et al., 1995, 1997; Tandon et al., 2008).

In experiments involving recordings from the depths of sulci, particularly in the case of animals with deafferentations, a recording site could be nonresponsive because of the electrode tip not being in the vicinity of layer 4 . Although in the figures we show only those recording sites that were judged to be in the middle layers of the cortex, the possibility of overestimating nonresponsive sites cannot be entirely ruled out.

\section{Results}

We determined somatotopic organization of areas S2, PV, and 3b after long-term, unilateral lesions of the dorsal columns of the spinal cord at cervical levels in three adult macaque monkeys. These areas were also mapped in two normal monkeys. We de-

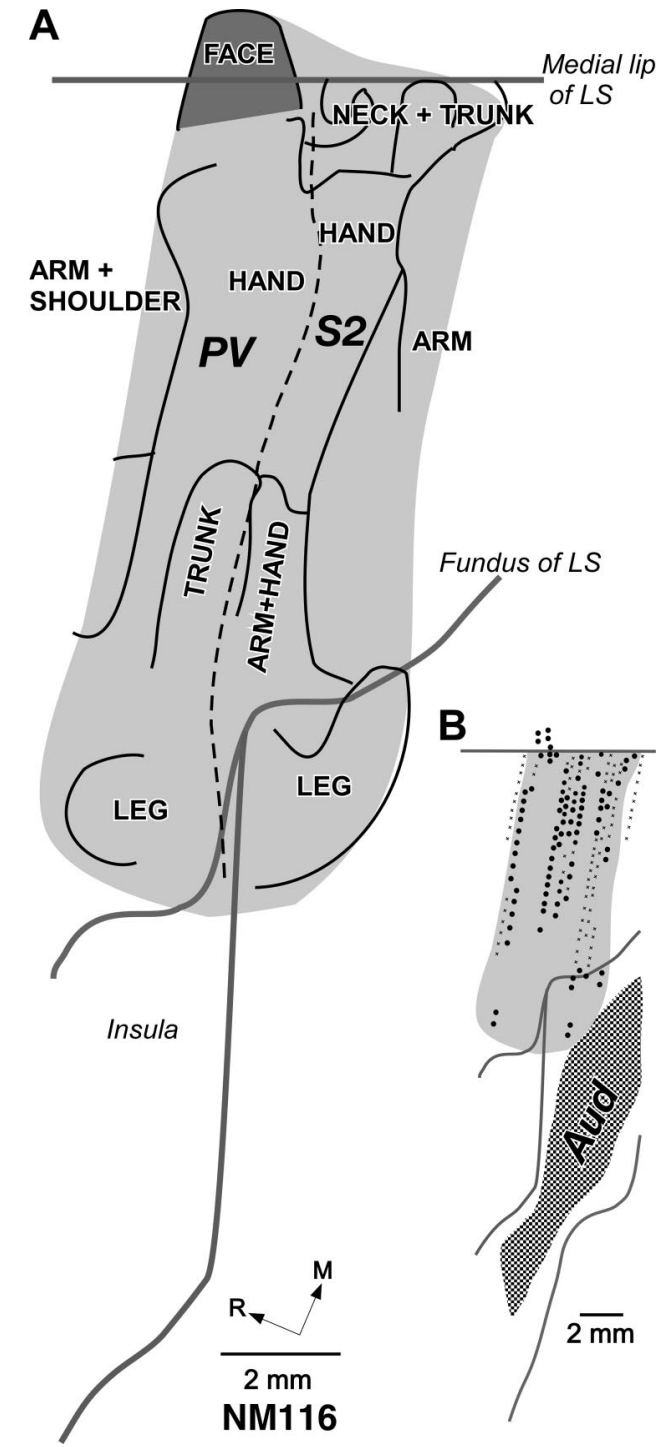

Figure 2. Organization of areas $S 2$ and PV in a normal adult macaque monkey, NM116. $A$, Areas S2 and PV (shaded in light gray) have a medial-to-lateral sequential representation of the face, hand/arm, trunk, and lower limb. The border between area S2 and area PV is marked by a dashed line. The thick gray lines depict the lips and the fundus of various sulci (Fig. 1C). The thin black lines mark boundaries between representations of different body parts, as labeled. The region shaded in dark gray corresponds to the face representation. $\boldsymbol{B}$, Reconstruction of the cortical surface as in $\boldsymbol{A}$ showing recording sites. The large dots mark the sites in which neurons responded to tactile stimulation, and small dots mark nonresponsive sites. The stippled area in the lower bank of the lateral sulcus is the core region of the auditory cortex (Aud) that was delineated from sections stained for acetylcholine esterase. Conventions are as for Figure 1.

scribe below changes in the organization of areas S2 and PV and relate these changes to reorganization in area $3 \mathrm{~b}$.

\section{Normal organization of areas S2 and PV}

Areas S2 and PV of macaque monkeys are located in the upper bank of the lateral sulcus (Fig. 1), lateral to the body representations in the anterior parietal areas 1 and 2 (Burton et al., 1995; Krubitzer et al., 1995), and may extend rostrally up to area $3 b$ (Disbrow et al., 2003; Padberg et al., 2009).

We mapped areas S2 and PV in two normal monkeys, NM14 and NM116. The observed somatotopy (Fig. 2) was similar to the one reported previously (Burton et al., 1995; Krubitzer et al., 1995). The hand representation was in the middle. It was bordered rostrally and caudally by representations of the arm in areas 
PV and S2, respectively. Representations of the trunk and lower limb were lateral to the hand representation. The head and the face representations were medial-most, near the lip of the lateral sulcus. In normal monkeys, there were no responses to touch on the face in regions in which inputs from other body parts were present (Krubitzer et al., 1995). In both monkeys, neurons at all recording sites in S2 and PV responded vigorously to touch or light taps on the skin.

\section{Reorganization of areas $3 \mathrm{~b}$ and S2/PV} after dorsal column transections We mapped areas S2 and PV, as well as area $3 \mathrm{~b}$ in three macaque monkeys with unilateral lesions of the dorsal columns $>1$ year after the lesion. In these three monkeys, a total of 589 recording sites was judged to be in the middle layers of areas S2 and PV. This mapping density compares well with the previous mapping studies of these areas [584 recording sites in five monkeys (Pons et al., 1987a); 181 recording sites in two monkeys (Pons et al., 1988); 491 recording sites in areas S2 and PV in three monkeys (Krubitzer et al., 1995)].

Areas S2 and PV are distinguished primarily based on the progression of RFs on the body surface as the electrode is moved in a rostral to caudal direction (Burton et al., 1995; Krubitzer et al., 1995; Disbrow et al., 2003). Although we could easily approximate the boundary between areas S2 and PV in normal monkeys (Fig. 2), we could not make this distinction in monkeys with lesions because the somatotopy, neuronal responses, and the RFs were not normal. In lesioned monkeys, we refer to the somatotopic representation in the lateral sulcus as S2/PV. Results from each monkey are described below.

\section{Monkey LM31}

This monkey was mapped 20 months after lesion of the dorsal columns on left side (Fig. 3). The lesion at C3 spinal level was complete except for minor sparing of the ventromedial part of the dorsal columns. There was also damage to the dorsal horn and parts of the ventral horn (Fig. 3C).

Somatotopy in area $3 \mathrm{~b}$ of the contralesional right hemisphere of this monkey showed extensive reorganization. We found responses to touch on the face in the region in which inputs from the hand were expected (Fig. 3A). Face-responsive neurons were found up to 9.3 $\mathrm{mm}$ medial to the normal hand-face border, which was the medial-most part of area $3 \mathrm{~b}$ that was mapped. For determining the extent of face expansion, the normal prelesion handface border was placed near the tip of the intraparietal sulcus (Florence and Kaas, 1995; Jain et al., 2008).
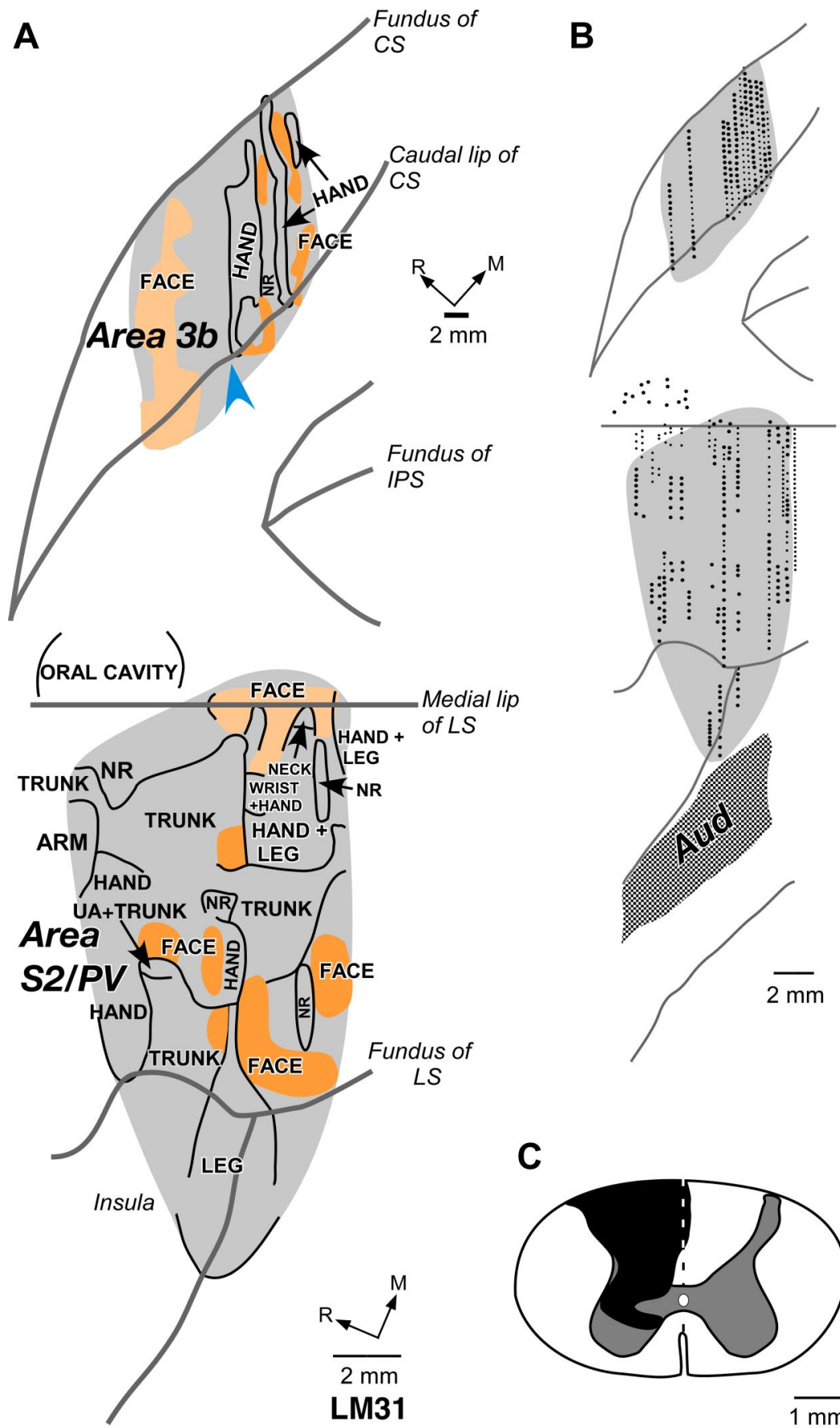

Figure 3. Reorganization of areas 3b, S2, and PV in monkey LM31. A, Flattened view of the cortex that includes area $3 b$ and S2/PV (Fig. 1). The normal face representation in area $3 \mathrm{~b}$ and the surrounding cortex, and area S2/PV is shown in pink. Note the expansion of the face representation in area $3 b$ as well as S2/PV (regions shaded in orange). The expected normal hand-face border in area $3 \mathrm{~b}$ is marked by a blue arrowhead. Note that, in this view, the caudal lip of the central sulcus is toward the bottom part of the figure. $\boldsymbol{B}$, Location of recording sites in area $3 \mathrm{~b}$ and S2/PV. C, Extent of the spinal cord lesion shown in a coronal view. The vertical dashed line marks the midline. NR, No response; UA, upper arm. For other conventions, see Figure 1.

Because of sparing of some portions of the dorsal columns, we also found responses to touch on the hand in area 3 b (Fig. 3). Receptive fields on the hand were large and confined to the glabrous palm, back of the hand, and proximal part of the dorsal digit 1 (D1). No response could be evoked by stimulation of the hairs (Schneider, 1990). Neuronal responses to stimulation of the hand were not as vigorous as in area $3 b$ of normal monkeys. In the expected face region of area $3 b$, lateral to the tip of the intraparietal sulcus, the responses were normal. In this region, the RFs 
A

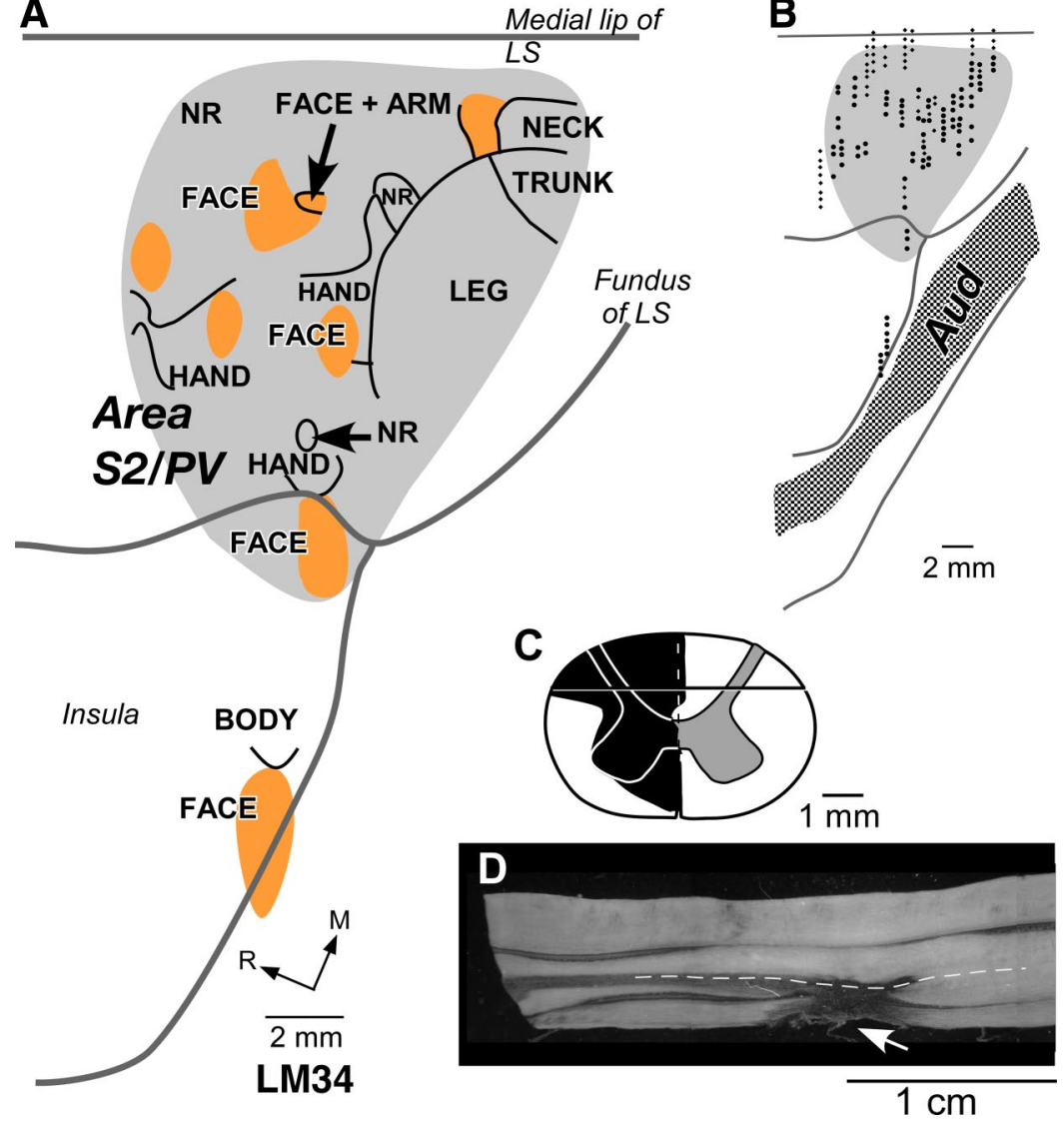

Figure 4. Reorganization of areas $S 2$ and PV in monkey LM34. $A$, Note the expanded representation of the face throughout S2/PV (region shaded in orange). $\boldsymbol{B}$, Location of the recording sites. $\boldsymbol{C}$, The extent of the spinal cord lesion shown in a coronal view. Outline of the gray matter on the left side is shown for reference. The horizontal line marks the location from where the section shown in $\boldsymbol{D}$ was taken. $\boldsymbol{D}$, A composite of dark-field photomicrographs of a horizontal section of the spinal cord showing the lesion site (arrow). The dashed line marks the midline. Conventions are as for Figures 1 and 3.

were small and neurons responded to light cutaneous touch or movement of the hairs on the face (Nelson et al., 1980; Pons et al., 1987b). All these features of area 3b organization are similar to those reported previously for monkeys with partial lesions of the dorsal columns (Jain et al., 1997, 2008).

In area $\mathrm{S} 2 / \mathrm{PV}$ of the contralesional right hemisphere of this monkey, there were only few recording sites in which neurons responded to the stimulation of the hand (Fig. 3A). Responses were evoked by stimulation of the deep receptors by taps or hard taps on the hand. There were no responses to cutaneous touch or light taps. Receptive fields of the neurons at these sites were confined to the thenar pad, glabrous skin of D1, back of the hand, and dorsal D1. The hand representation in S2/PV was much smaller compared with that in normal monkeys.

At many recording sites in $\mathrm{S} 2 / \mathrm{PV}$, neurons responded to touch on the face (Fig. 3A). One of the face-responsive regions was at the normal expected location near the medial lip of the lateral sulcus. However, neurons responding to stimulation of the face were also present in the regions of the hand, arm, trunk, and leg representations (Fig. 3A). Neurons responding to touch on the face at these locations were not seen in normal monkeys (Fig. 2) (Robinson and Burton, 1980; Krubitzer et al., 1995). At some recording sites, neurons did not respond to the stimulation of any body part. The data show that, after deafferentation by lesions of the dorsal columns, there was expansion of the face inputs into the hand and the trunk regions of area S2/PV. Neurons responding to touch on the face were observed as far as 11 $\mathrm{mm}$ from the medial border of area S2/ $\mathrm{PV}$. The total mediolateral extent of area $\mathrm{S} 2 / \mathrm{PV}$ in this monkey was $14 \mathrm{~mm}$.

Neurons in area S2/PV of this monkey also responded to stimulation of the arm, lower limb, and trunk at normal locations for these representations. Response properties of neurons at these sites and their RFs were similar to those seen in normal monkeys. However, at a few sites medial to the hand region, there were responses to taps on the dorsal upper trunk, indicating a somewhat larger representation of the trunk compared with normal monkeys.

\section{Monkey LM34 \\ Monkey LM34 was mapped 13 months after} unilateral lesion of the dorsal columns on the left side at C3-C4 level (Fig. 4). The lesion was complete with slight sparing of the medioventral dorsal columns. Ipsilateral gray matter, mediodorsal parts of the ventral funiculus, parts of the dorsolateral funiculus, and the medial-most part of the lateral funiculus were also damaged (Fig. 4C,D).

In area $3 \mathrm{~b}$ of this monkey, as for monkey LM31, there was large-scale expansion of the face representation into the deafferented hand region (data not shown). Neurons responding to tactile stimulation of the face were present throughout the hand region. The face inputs were observed as far as 10 $\mathrm{mm}$ medial from the normal hand-face border. At three recording sites, we also found neurons responding to taps on the hand. Two of these RFs were on the thenar pad and one covered the entire palm. At two additional recording sites, neurons responded to hard taps on the hand. These inputs likely reflect minor sparing of fibers of the dorsal columns.

In area $\mathrm{S} 2 / \mathrm{PV}$ of this monkey, as for monkey LM31, neurons responded to tactile stimulation of the face at many abnormally located recording sites. These recording sites were located in the region in which responses to touch on the hand and other body parts are normally seen (Fig. 4A). At some of the recording sites, dual RFs on face plus arm were also observed. In this monkey, the face expansion was $12 \mathrm{~mm}$ from the medial border of S2/PV. The lateral-most face-responsive region shown in Figure 4 might be in the proposed ventral somatosensory (VS) area (Cusick et al., 1989; Krubitzer et al., 1995).

In area $\mathrm{S} 2 / \mathrm{PV}$, we also found responses to touch on the hand at a few recording sites. Receptive fields of neurons at these sites were confined to the thenar pad. We did not encounter any neurons with RFs on the digits or other parts of the hand. At the remaining sites in area S2/PV, neuronal responses were evoked by stimulation of the trunk or the lower limb. The response properties of these neurons and their RFs were as in normal monkeys. There were few recording sites in which neurons did not respond to stimulation of any body part. The normal face region of this monkey was not mapped.

Monkey LM01

Monkey LM01 was mapped after lesion of the dorsal columns on the left side at C4 level (Fig. 5). The lesion damaged the medial 
part of the dorsal columns. Dorsal column fibers in the lateral half were spared, with additional minor sparing in the ventral most region of the medial part (Fig. 5D). The damage extended to the dorsal parts of the ventral horn and the intermediate gray matter. Consistent with considerable sparing of the dorsal columns, we found many recording sites with responses to touch on the hand in area $3 \mathrm{~b}$ of this monkey (Fig. 5A). Receptive fields of the neurons were distributed over the entire glabrous skin of the hand except ulnar half of the palm. However, neurons responded to stimulation of deep receptors by taps, and not to light cutaneous touch. The receptive fields were large, covering an entire digit, a complete phalange of a digit, or a palmar pad. Although an overall somatotopy in the hand region, with representations of D1 to D5 in a lateral-tomedial sequence was clear, it was not as well defined as in normal monkeys.

Despite considerable sparing of the dorsal column fibers, there was large-scale expansion of the face inputs into the hand region of area $3 \mathrm{~b}$ (Fig. 5A). Face inputs were observed as far as $7.6 \mathrm{~mm}$ medial from the normal hand-face border. Receptive fields of neurons in the deafferented hand region were on the hand, the face, or covered parts of both the face and hand. All these features of organization of area $3 \mathrm{~b}$, including presence of dual RFs, are similar to those reported previously in monkeys with partial lesions of the dorsal columns (Jain et al., 1997, 2000, 2008). The normal face representation, lateral to the tip of the intraparietal sulcus, was unaltered.

In area $\mathrm{S} 2 / \mathrm{PV}$ of this monkey, somatotopy was more normal-like (Fig. 5B) compared with the other two monkeys with lesions. There was a well defined representation of the hand in the middle; the neuronal responses to taps on the hand were vigorous and the RFs were observed over a much larger part of the hand, which included digits and the thenar pad. The hand representation was bordered by arm representations rostrally and caudally. Medial to the hand representation, near the upper lip of the lateral sulcus, neurons responded to touch on the face as expected. Laterally, inputs from the leg were observed at normal locations. At few recording sites, we also found neurons with RFs on the tail and proximal leg. Neuronal responses and the sizes of RFs in the trunk and the lower limb regions were normal (Robinson and Burton, 1980; Krubitzer et al., 1995). Neurons in the lateral-most part of the mapped region had large and complex RFs that extended over the contralateral arm as well as leg. This region corresponds to the location of the proposed area VS (Robinson and Burton, 1980; Cusick et al., 1989; Krubitzer et al., 1995).

Although the hand representation in S2/PV was normal-like in this monkey, within the forelimb representation we found
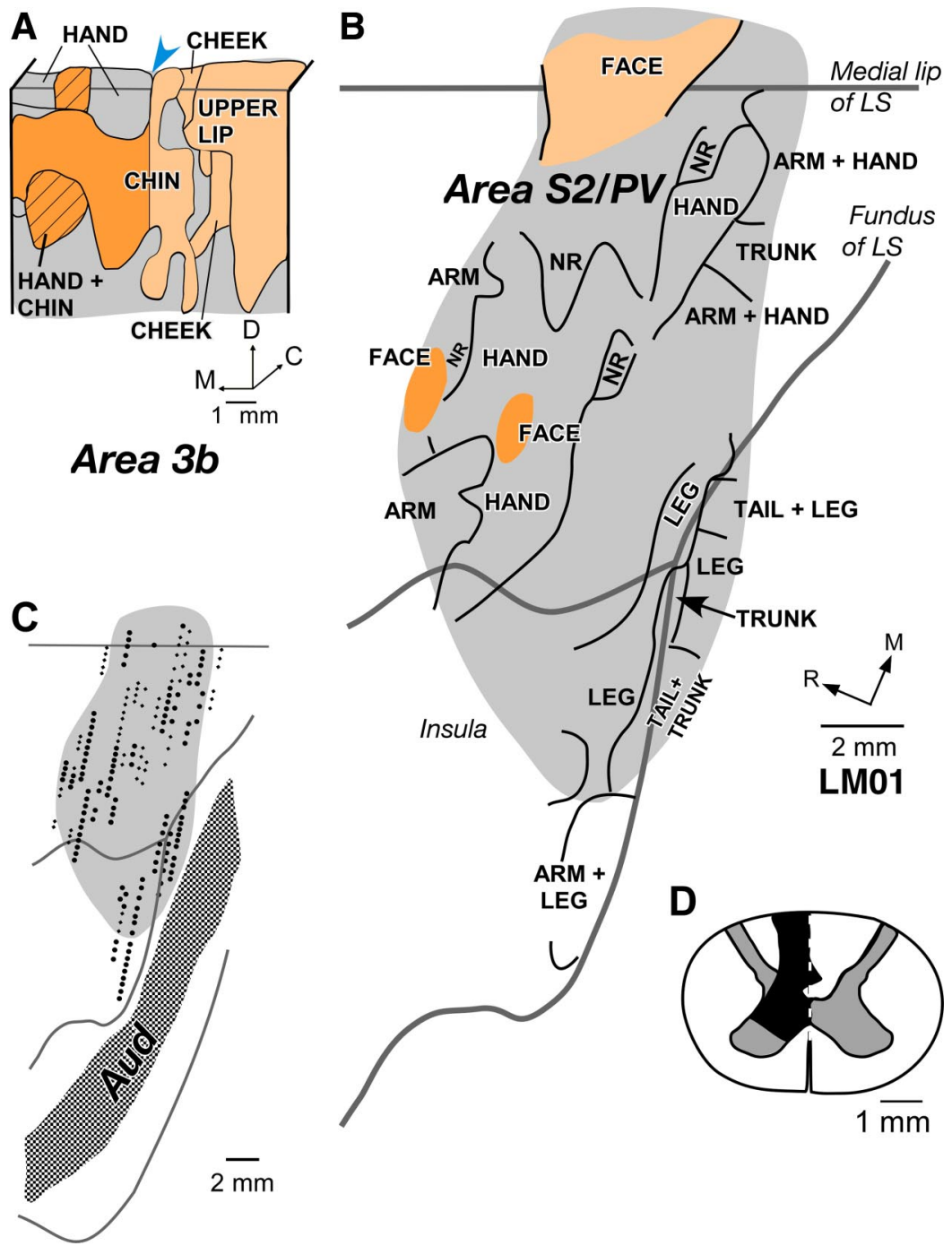

Figure 5. Reorganization of areas $3 \mathrm{~b}$ and S2/PV in monkey LM01. A, A reconstruction of the posterior bank of the central sulcus showing the somatotopic map in area $3 \mathrm{~b}$ and the surrounding cortex. Note the expanded face representation (shaded in orange) de ferented hand region. Normal location of the hand-face border is marked by a blue arrowhead on the top. The regions Note large regions with responses to tactile stimulation of the hand, which were present dorsal columns. C, Location of all the recording sites in area S2/PV. D, Extent of the lesion shown in a coronal view of the spinal cord. Conventions are as for Figures 1 and 3.

responses to touch on the face at many recording sites. The face inputs had, therefore, expanded into the hand region even in this monkey with considerable sparing of the dorsal columns. The lateral-most recording site in which neurons responded to touch on the face was $8 \mathrm{~mm}$ from the medial border of area S2/PV.

\section{Face-receptive fields in the reorganized parts of area $3 b$ and S2/PV}

We compared sources of face inputs to the deafferented hand regions of areas $3 \mathrm{~b}$ and $\mathrm{S} 2 / \mathrm{PV}$ in monkeys with spinal cord lesions. In the expanded face representation in area $3 \mathrm{~b}$ of all the three monkeys, all the RFs were on the chin (Fig. $6 A, B$ ). Similarly, in area S2/PV, the majority $(70 \% ; n=57)$ of the RFs in the reorganized cortex were either entirely or partly on the chin (Fig. $6 C, D)$. In S2/PV of monkey LM01, all such RFs were on the chin. In monkey LM34, $18.2 \%$ of the RFs were entirely on the chin, 
A

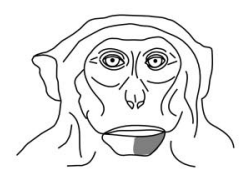

B
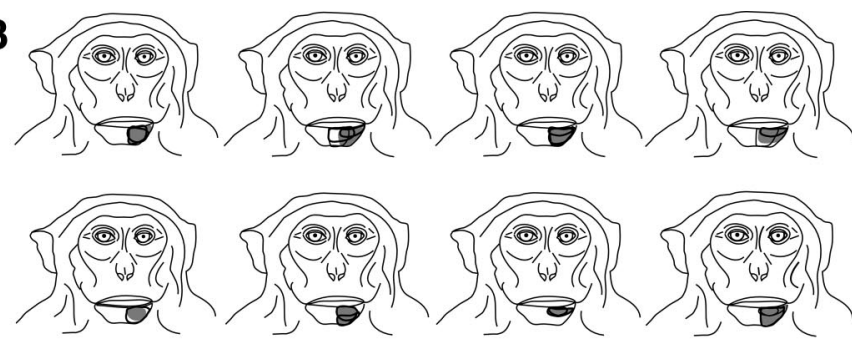

C

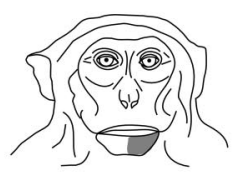

D

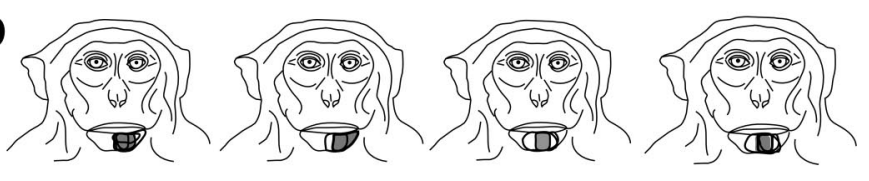

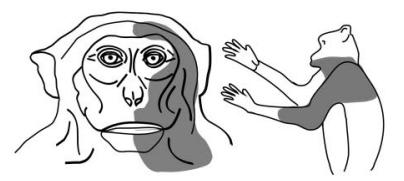
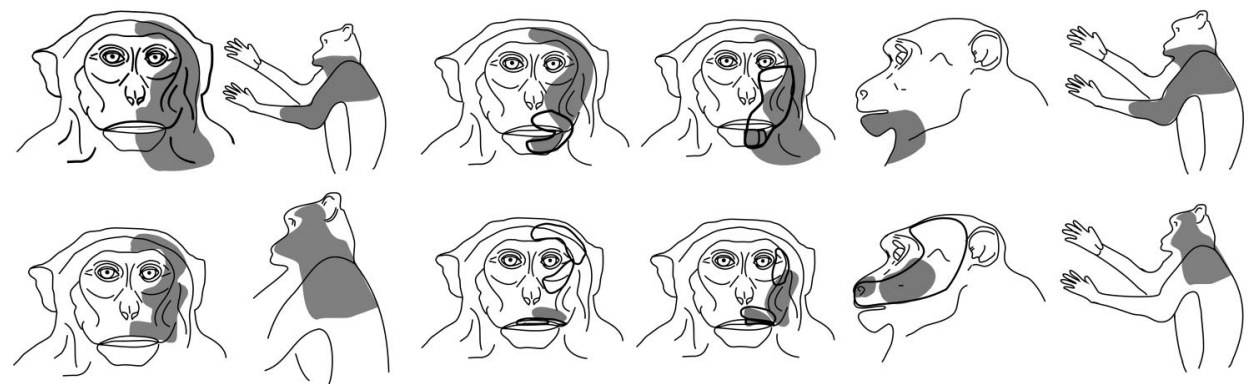

Figure 6. Comparison of receptive fields of neurons in the expanded face representation in areas $3 \mathrm{~b}$ and S2/PV. $\boldsymbol{A}$, Region of the face covered by receptive fields in the deafferented parts of area $3 \mathrm{~b}$ of all the three monkeys with dorsal column lesions. Note that, in area $3 \mathrm{~b}$, only chin inputs expand. $\boldsymbol{B}$, Figurines showing different kinds of receptive fields on the chin in the deafferented parts of area $3 \mathrm{~b}$ of the three monkeys. If the same receptive field was encountered multiple times, it is shown only once. $\boldsymbol{C}$, Region of the face covered by all the receptive fields in the reorganized part of area S2/PV in all the three monkeys. The top figurine shows combined receptive fields that were entirely on the chin; middle figurines, the composite of receptive fields that were on the chin but also extended to the other parts of the face; and bottom figurines, the receptive fields that excluded the chin. $\boldsymbol{D}$, Different kinds of receptive fields on the face in the reorganized parts of area $\mathbf{S 2}$ /PV in the three monkeys. Receptive fields at the same location encountered more than once are not shown separately.

$59.1 \%$ covered chin as well as other parts of the face or occiput, whereas the remaining $22.7 \%$ did not include chin. Finally, in monkey LM31, $58.6 \%$ of the RFs were on the chin, whereas the remaining $41.4 \%$ did not include chin. Therefore, mostly chin inputs expanded in both area $3 \mathrm{~b}$ and S2/PV; however, in S2/PV of two of the lesioned monkeys, the expanded face representation also included other parts of the face.

\section{Receptive fields on the hand in area $3 \mathrm{~b}$ and S2/PV}

We compared the hand representations in area $3 \mathrm{~b}$ and S2/PV of the lesioned monkeys. As described above, the three lesioned monkeys had different extents of hand representations remaining in these areas depending on the extent of sparing of the dorsal column fibers. In area $3 \mathrm{~b}$ of the lesioned monkeys, the hand RFs were larger compared with RFs in area $3 \mathrm{~b}$ of normal monkeys (Fig. $7 A, B$ ), whereas in areas $\mathrm{S} 2$ and PV, the RFs were of similar size in both normal and lesioned animals (Fig. 7C,D). To compare across different monkeys, we quantified sizes of RFs on the hand in area $3 \mathrm{~b}$ and S2/PV. RFs for all monkeys were scaled to a standard diagram of the hand, and the area covered by each RF was measured using Canvas software. Our statistical analysis showed that RF sizes in the two normal monkeys were not significantly different ( $p=0.072$ for area $3 b$, monkeys NM24 and NM36; $p=0.188$ for S2/PV, monkeys NM14 and NM116; Mann-Whitney rank sum test). We, therefore, averaged data from the normal monkeys.

Comparisons between normal and lesioned monkeys showed that, in area $3 \mathrm{~b}, \mathrm{RFs}$ in the lesioned monkeys were significantly larger compared with those in the normal monkeys (MannWhitney rank sum test). Figure $7 E$ shows that the average size of the RFs in area $3 \mathrm{~b}$ of monkey LM31 was 15.8 times larger than normal ( $p<0.001)$; in monkey LM34, it was 17 times larger $(p<0.05)$; and in monkey LM01, it was 6.1 times larger $(p<$ $0.001)$. However, RF sizes in areas S2 and PV between the lesioned and the normal monkeys were not significantly different (Fig. $7 F$ ). In monkey LM31, the average RF size was 1.2 times that of normal monkeys ( $p=0.531)$; in monkey LM34, it was 0.85 times $(p=0.624)$; and in monkey LM01, the average RF size was 0.90 times that in normal monkeys $(p=0.612)$. Thus, the large RFs on the hand in areas S2 and PV do not become larger after deafferentations.

\section{Discussion}

Our results show that higher somatosensory areas S2 and PV of macaque monkeys undergo large-scale reorganization after deafferentation by unilateral lesions of the dorsal columns of the spinal cord. Reorganization in areas S2 and PV is similar to that seen in area $3 b$, the primary somatosensory area. We discuss our results in the context of plasticity of the adult somatosensory system and its implications for understanding perceptual consequences of the brain reorganization.

\section{Reorganization at multiple levels in the somatosensory system}

Large-scale reorganization of area $3 b$ resulting in expansion of the face inputs into the deafferented hand region takes place after transection of the cervical dorsal columns of the spinal cord (Jain et al., 1997, 2000, 2008) or cutting of the dorsal roots from C2 to T4 (Pons et al., 1991). Similar expansion of the face representation takes place in the VP nucleus of thalamus (Pons et al., 1991; Jones and Pons, 1998; Jain et al., 2008) (but see Graziano and Jones, 2009). Reorganization at multiple levels, the somatosen- 
sory cortex, the thalamus, and the brainstem nuclei, is also seen after more limited deafferentations. For example, intact radial nerve inputs expand after cutting of the median and ulnar nerves to the hand (Garraghty and Kaas, 1991a; Garraghty et al., 1994; Xu and Wall, 1999; Churchill et al., 2001), and inputs from the stump expand after amputation of a limb (Florence et al., 2000). Topographic reorganization in the primary somatosensory cortex and the VP nucleus of thalamus is also seen in humans after spinal cord injuries or amputations (Lenz et al., 1987, 1994; Davis et al., 1998; Dostrovsky, 1999; Grüsser et al., 2004) and in other mammalian species after chronic or acute deafferentations (Dostrovsky et al., 1976; Devor and Wall, 1981; Wall and Cusick, 1984; Calford and Tweedale, 1988; Byrne and Calford, 1991; Turnbull and Rasmusson, 1991; Pettit and Schwark, 1993; Rasmusson et al., 1993; McCandlish et al., 1996; Faggin et al., 1997). Here, we show that reorganization of the adult brain is more extensive and also takes place in higher somatosensory areas S2 and PV (Fig. 8). Although areas S2 and PV were not distinguished in the lesioned monkeys (see Results), the presence of the expanded face representations in the entire rostrocaudal extent of the somatosensory region of the lateral sulcus indicates that both S2 and PV undergo reorganization.

\section{Sources of altered inputs to S2/PV}

There are two major sources of inputs to area S2 and PV in primates. Area 3b provides direct cortical inputs that terminate in layer 4 (Friedman et al., 1986; Burton et al., 1995; Disbrow et al., 2003). In addition, areas S2 and PV get direct thalamic inputs from the ventroposterior inferior (VPI) nucleus (Friedman and Murray, 1986; Stevens et al., 1993; Disbrow et al., 2002). VPI in turn is a major recipient of inputs from the spinothalamic pathways (Apkarian and Hodge, 1989; Rausell and Jones, 1991; Kaas, 1993). Since spinothalamic tracts were not damaged in our monkeys, these inputs from the forelimb and other body parts would continue to be relayed to areas S2 and PV. However, this did not prevent large-scale expansion of the face representation in S2 and PV. Since the only source of altered inputs to area S2 (and PV) is area 3b, we propose that the reorganization in areas S2 and PV reflects changes in area $3 \mathrm{~b}$. This proposal is in conformity with the previous reports that area $3 \mathrm{~b}$ provides driving inputs to S2, and perhaps PV (Pons et al., 1987a, 1992; Garraghty et al., 1990b) (but see Zhang et al., 1996). Hierarchical nature of processing in the somatosensory system, which places area S2 upstream of area 3b, has been suggested before (Friedman et al., 1986; Pons et al., 1987a, 1992; Kaas, 2004).

In our monkeys with lesions, areas S2 and PV remained responsive to tactile stimulation of the trunk and lower limb. It has been shown previously that inputs from the trunk and lower limb
B
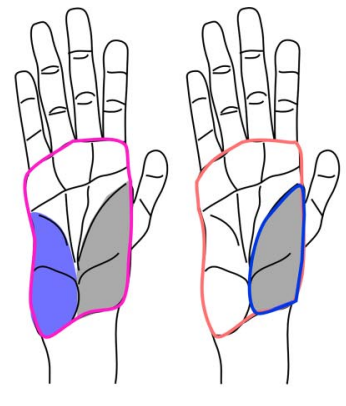

LM34

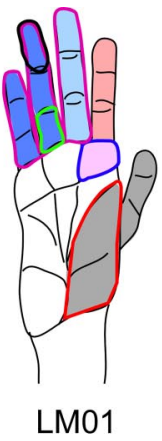

D
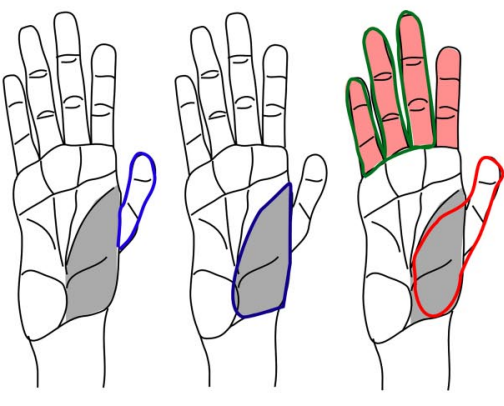

LM34
LM01
$\mathbf{F}$

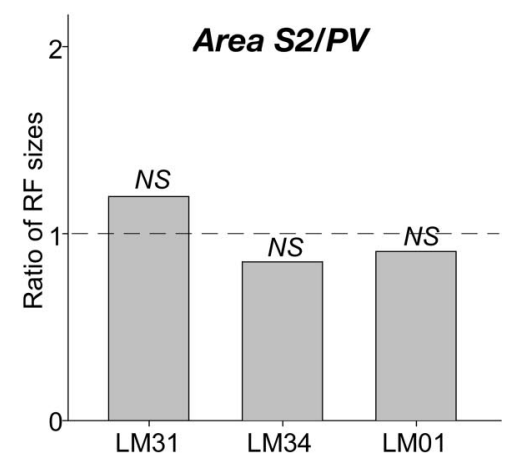

Figure 7. Receptive fields on the hand in areas $3 \mathrm{~b}$ and $\mathrm{S} 2 / \mathrm{PV}$ of normal and lesioned monkeys. $\boldsymbol{A}, \boldsymbol{B}$, Examples of receptive fields on the hand in area $3 \mathrm{~b}$ of two normal monkeys $(\boldsymbol{A})$ and three monkeys with lesions of the dorsal columns $(\boldsymbol{B})$. Note that the receptive fields in the lesioned monkeys are large. $\boldsymbol{C}, \boldsymbol{D}$, Receptive fields of neurons in areas $\boldsymbol{S} 2$ and PV of normal monkeys $(\boldsymbol{C})$ and normal monkeys for area $3 \mathrm{~b}$ and area S2/PV, respectively. The dashed lines correspond to the ratio of 1 . Note different scales for the $y$-axes in $\boldsymbol{E}$ and $\boldsymbol{F} .{ }^{*} p<0.05{ }^{* * *} p<0.001$; NS, not significant.

continue to activate neurons at their normal somatotopic locations in area $3 \mathrm{~b}$ even in monkeys with complete lesions of the dorsal columns (Jain et al., 2008), which could be attributable to activation by inputs via the dorsolateral funiculus. Area $3 \mathrm{~b}$ is therefore the likely source of activating trunk and lower limb inputs to S2/PV of the lesioned monkeys in the present study. In addition, we also checked for responses to the stimulation of the ipsilateral hand in the two normal and one lesioned monkey. In $\mathrm{S} 2 / \mathrm{PV}$ of the lesioned monkey, responses of neurons to the stimulation of the ipsilateral hand were as in normal animals (Robinson and Burton, 1980). Receptive fields were found over nearly the entire ipsilateral hand, whereas those on the contralateral hand were restricted to the inputs via the spared fibers as described above.

\section{Nature of reorganization at multiple levels}

Previous reports show that the nature of reorganization in both the VP nucleus of thalamus and area $3 \mathrm{~b}$ is mostly similar. In both 


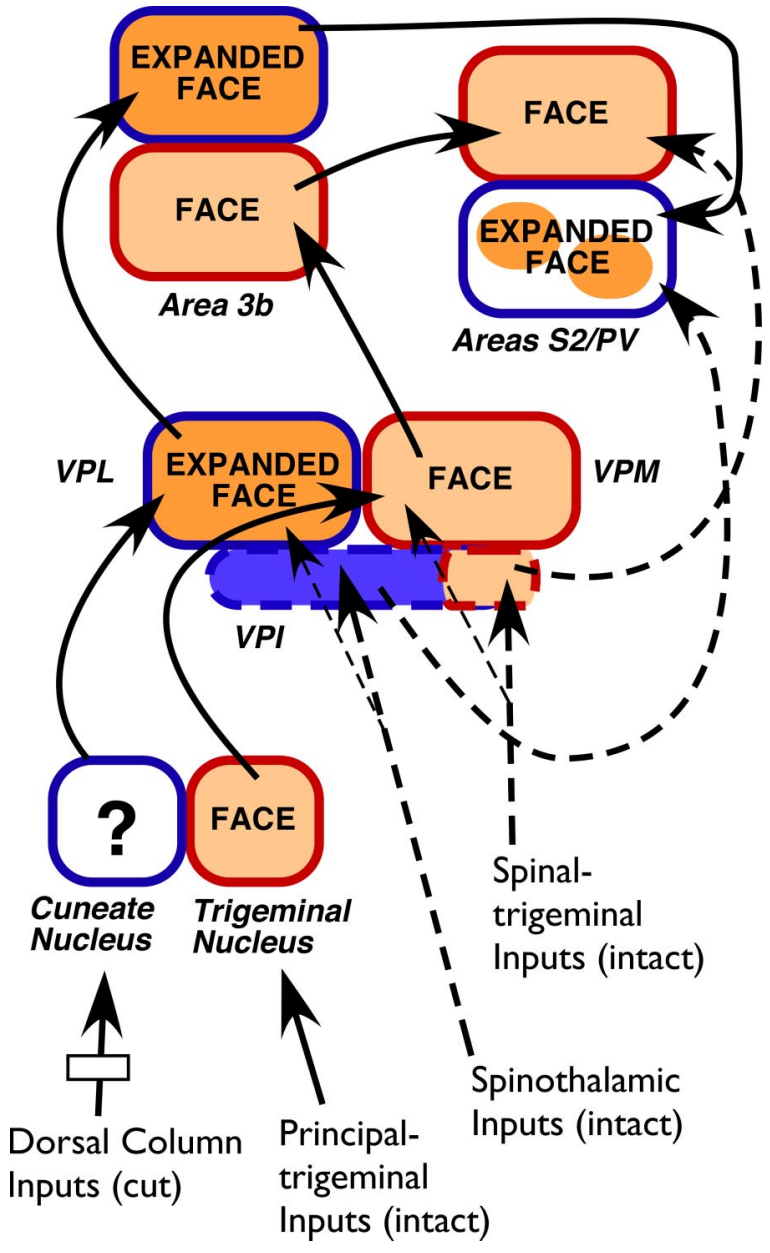

Figure 8. A schematic showing large-scale reorganization at different levels in the somatosensory system after lesions of the dorsal columns. Expansion of the face inputs into the hand region has been observed in the VP nucleus of thalamus, area $3 \mathrm{~b}$, and areas $\mathrm{S} 2$ and PV. A nearly complete "filling in" of the deafferented regions was observed in the VP nucleus and area 3b, whereas the expansion was patchy in areas S2 and PV. It is presently not known whether there is a similar expansion of the face inputs into the cuneate nucleus of the brainstem, although growth of afferents from the trigeminal nucleus into the cuneate nucleus has been shown (see Discussion). The outline color indicates the expected representation (blue, hand; red, face) and the fill color shows the observed representation after lesions of the dorsal columns (pink, normal face; orange, expanded face). The solid arrows show lemiscal inputs; the dashed arrows indicate the intact spinothalamic inputs via the VPI nucleus and the equivalent inputs from the face. The thin dashed lines indicate weaker inputs. The overall convergence and divergence of the inputs is much more extensive than shown here. For additional details and references, see Discussion.

area $3 \mathrm{~b}$ and the VP nucleus, intact radial nerve inputs expand after transection of the median and ulnar nerves (Wall et al., 1986; Garraghty et al., 1994), inputs from the skin of the stump expand after limb amputations (Florence and Kaas, 1995; Davis et al., 1998; Lenz et al., 1998; Dostrovsky, 1999), and inputs from the chin expand after lesions of the dorsal columns of the spinal cord and dorsal root transections (Pons et al., 1991; Jain et al., 1997, 2008; Jones and Pons, 1998). In the present study also, we observed expansion of the chin inputs at majority of the recording sites in areas S2 and PV. Expansion of inputs from the same body part at different somatosensory processing levels suggests that reorganization at higher levels could be a reflection of the reorganization at a downstream level. It has been proposed that reorganization in the VP nucleus of thalamus and area $3 \mathrm{~b}$ reflects reorganization in the cuneate nucleus of the brainstem, which could be attributable to growth of intact chin afferents from the adjacent trigeminal nucleus (Sengelaub et al., 1997; Kaas et al., 1999; Jain et al., 2000). These changes could be propagated further into areas S2 and PV.

We also observed differences in the nature of RFs in the reorganized primary and secondary somatosensory areas. For example, in area $3 b$, expansion of only the chin inputs was seen, whereas in area S2 and PV at $\sim 30 \%$ of the recording sites, the expanded face representation did not include chin inputs. Differences in the details of reorganization at different levels of neuraxis have been noted before. Jain et al. (2008) reported extensive expansion of the inputs from the occiput region into the deafferented hand region in the VP nucleus, but expansion of the occiput representations in the deprived hand region of area $3 \mathrm{~b}$ was restricted to its medial border. Florence et al. (2000) also noted differences between the reorganization in the VP nucleus and area $3 \mathrm{~b}$ of monkeys with limb amputations. Details of reorganization at each level differ, perhaps because of differences in local features such as divergence and convergence of inputs (Florence and Kaas, 1995; Jones and Pons, 1998; Rausell et al., 1998; Padberg et al., 2009), nature of horizontal connections (Manger et al., 1997; Jones, 2000), extent of modulation by feedback connections (Ergenzinger et al., 1998; Krupa et al., 1999; Parker and Dostrovsky, 1999), extent of postlesion axon withdrawal (Graziano and Jones, 2009), and local growth (Darian-Smith and Gilbert, 1994). For example, it is possible that convergent inputs from the expanded chin and occiput regions in area $3 \mathrm{~b}$ give rise to RFs overlapping both these body surfaces in S2/PV.

\section{Perceptual consequences of plasticity}

It has been proposed (Ramachandran et al., 1992; Jain, 2002) that plasticity provides an anatomical and neurophysiological substrate for referred phantom sensations that are experienced by patients with amputation of a limb (Melzack, 1971; Katz and Melzack, 1990; Ramachandran, 1993) or spinal cord injury (Sweet, 1975; Ettlin et al., 1980; Coderre et al., 1993). These phantom sensations often have a strong affective component (Ramachandran and Rogers-Ramachandran, 2000) and are known to trigger preinjury memories of position and sensations of the affected limb (Katz and Melzack, 1990). Abnormal output via reorganized S2 and PV could act as a trigger for such phantom sensations because somatosensory areas of the lateral parietal cortex project via the granular and dysgranular insular cortex to the amygdaloidal complex, and via perirhinal cortex to the hippocampal formation, structures that have a role in memory formation and mediating affective aspects of sensations (Mishkin, 1979; Friedman et al., 1986) (but see Disbrow et al., 2003). Interestingly, Ramachandran (1993) reports that, in patients with amputations of an arm, phantom sensations are evoked by touch on a large part of the face that includes the chin, upper lip, and side of the face, the gamut of RFs on the face in the reorganized S2 and PV observed by us.

Melzack (1990) proposed that neuromatrix, a diffuse network of neurons, which remains mostly intact after spinal or peripheral injuries, triggers phantom sensations. After injury to the dorsal columns of the spinal cord, the hand region of $\mathrm{S} 2 / \mathrm{PV}$ receives somatotopically inappropriate face inputs from the reorganized area $3 \mathrm{~b}$ as well as normal hand inputs from VPI of thalamus. The abnormal inputs would create a conflict in the readout from $\mathrm{S} 2 / \mathrm{PV}$ if somatotopy in these areas remained normal, resulting in a touch on the face being perceived as a touch on the hand. The present results suggest that location of such a trigger for phantom sensations is likely to be upstream of cortical areas S2 and PV since these areas get reorganized. Alternatively, intact corticospi- 
nal efferents from the somatosensory cortex (Murray and Coulter, 1981) could provide a direct mismatch between inputs and outputs. Motor output from the primary motor cortex also remains normal after transection of the dorsal columns $(\mathrm{N}$. Kambi, S. Tandon, H. Mohammed, L. Lazar, and N. Jain, unpublished observations). Such mismatches (Guillery, 2005; Reilly and Sirigu, 2008) could also act as a perceptual trigger for phantom sensations.

\section{References}

Apkarian AV, Hodge CJ (1989) Primate spinothalamic pathways: III. Thalamic terminations of the dorsolateral and ventral spinothalamic pathways. J Comp Neurol 288:493-511.

Bromage PR, Melzack R (1974) Phantom limbs and the body schema. Can Anaesth Soc J 21:267-274.

Burton H, Fabri M, Alloway K (1995) Cortical areas within the lateral sulcus connected to cutaneous representations in areas $3 \mathrm{~b}$ and $1:$ a revised interpretation of the second somatosensory area in macaque monkeys. J Comp Neurol 355:539-562.

Byrne JA, Calford MB (1991) Short-term expansion of receptive fields in rat primary somatosensory cortex after hindpaw digit denervation. Brain Res 565:218-224.

Calford MB, Tweedale R (1988) Immediate and chronic changes in responses of somatosensory cortex in adult flying-fox after digit amputation. Nature 332:446-448.

Chen R, Cohen LG, Hallett M (2002) Nervous system reorganization following injury. Neuroscience 111:761-773.

Churchill JD, Arnold LL, Garraghty PE (2001) Somatotopic reorganization in the brainstem and thalamus following peripheral nerve injury in adult primates. Brain Res 910:142-152.

Coderre TJ, Katz J, Vaccarino AL, Melzack R (1993) Contribution of central neuroplasticity to pathological pain: review of clinical and experimental evidence. Pain 52:259-285.

Cusick CG, Wall JT, Felleman DJ, Kaas JH (1989) Somatotopic organization of the lateral sulcus of owl monkeys: area $3 \mathrm{~b}, \mathrm{~S}-\mathrm{II}$, and a ventral somatosensory area. J Comp Neurol 282:169-190.

Darian-Smith C, Ciferri M (2006) Cuneate nucleus reorganization following cervical dorsal rhizotomy in the macaque monkey: its role in the recovery of manual dexterity. J Comp Neurol 498:552-565.

Darian-Smith C, Gilbert CD (1994) Axonal sprouting accompanies functional reorganization in adult cat striate cortex. Nature 368:737-740.

Davis KD, Kiss ZH, Luo L, Tasker RR, Lozano AM, Dostrovsky JO (1998) Phantom sensations generated by thalamic microstimulation. Nature 391:385-387.

Devor M, Wall PD (1981) Plasticity in the spinal cord sensory map following peripheral nerve injury in rats. J Neurosci 1:679-684.

Disbrow E, Litinas E, Recanzone GH, Slutsky D, Krubitzer L (2002) Thalamocortical connections of the parietal ventral area (PV) and the second somatosensory area (S2) in macaque monkeys. Thalamus Relat Syst 1:289-302.

Disbrow E, Litinas E, Recanzone GH, Padberg J, Krubitzer L (2003) Cortical connections of the second somatosensory area and the parietal ventral area in macaque monkeys. J Comp Neurol 462:382-399.

Dostrovsky JO (1999) Immediate and long-term plasticity in human somatosensory thalamus and its involvement in phantom limbs. Pain Suppl 6:S37-S43.

Dostrovsky JO, Millar J, Wall PD (1976) The immediate shift of afferent drive to dorsal column nucleus cells following deafferentation: a comparison of acute and chronic deafferentation in gracile nucleus and spinal cord. Exp Neurol 52:480-495.

Dykes RW, Avendaño C, Leclerc SS (1995) Evolution of cortical responsiveness subsequent to multiple forelimb nerve transections: an electrophysiological study in adult cat somatosensory cortex. J Comp Neurol 354:333-344.

Ergenzinger ER, Glasier MM, Hahm JO, Pons TP (1998) Cortically induced thalamic plasticity in the primate somatosensory system. Nat Neurosci $1: 226-229$.

Ettlin TM, Seiler W, Kaeser HE (1980) Phantom and amputation illusions in paraplegic patients. Eur Neurol 19:12-19.

Faggin BM, Nguyen KT, Nicolelis MA (1997) Immediate and simultaneous sensory reorganization at cortical and subcortical levels of the somatosensory system. Proc Natl Acad Sci U S A 94:9428-9433.

Flor H, Elbert T, Knecht S, Wienbruch C, Pantev C, Birbaumer N, Larbig W, Taub E (1995) Phantom-limb pain as a perceptual correlate of cortical reorganization following arm amputation. Nature 375:482-484.

Florence SL, Kaas JH (1995) Large-scale reorganization at multiple levels of the somatosensory pathway follows therapeutic amputation of the hand in monkeys. J Neurosci 15:8083-8095.

Florence SL, Hackett TA, Strata F (2000) Thalamic and cortical contributions to neural plasticity after limb amputation. J Neurophysiol 83:31543159.

Friedman DP, Murray EA (1986) Thalamic connectivity of the second somatosensory area and neighboring somatosensory fields of the lateral sulcus of the macaque. J Comp Neurol 252:348-373.

Friedman DP, Murray EA, O’Neill JB, Mishkin M (1986) Cortical connections of the somatosensory fields of the lateral sulcus of macaques: evidence for a corticolimbic pathway for touch. J Comp Neurol 252: 323-347.

Garraghty PE, Kaas JH (1991a) Large-scale functional reorganization in adult monkey cortex after peripheral nerve injury. Proc Natl Acad Sci U S A 88:6976-6980.

Garraghty PE, Kaas JH (1991b) Functional reorganization in adult monkey thalamus after peripheral nerve injury. Neuroreport 2:747-750.

Garraghty PE, Florence SL, Kaas JH (1990a) Ablations of areas 3a and 3b of monkey somatosensory cortex abolish cutaneous responsivity in area 1. Brain Res 528:165-169.

Garraghty PE, Pons TP, Kaas JH (1990b) Ablations of areas 3b (SI proper) and $3 a$ of somatosensory cortex in marmosets deactivate the second and parietal ventral somatosensory areas. Somatosens Mot Res 7:125-135.

Garraghty PE, Hanes DP, Florence SL, Kaas JH (1994) Pattern of peripheral deafferentation predicts reorganizational limits in adult primate somatosensory cortex. Somatosens Mot Res 11:109-117.

Geneser-Jensen FA, Blackstad TW (1971) Distribution of acetyl cholinesterase in the hippocampal region of the guinea pig. I. Entorhinal area, parasubiculum, and presubiculum. Z Zellforsch Mikrosk Anat 114:460-481.

Graziano A, Jones EG (2009) Early withdrawal of axons from higher centers in response to peripheral somatosensory denervation. J Neurosci 29:3738-3748.

Grüsser SM, Mühlnickel W, Schaefer M, Villringer K, Christmann C, Koeppe C, Flor H (2004) Remote activation of referred phantom sensation and cortical reorganization in human upper extremity amputees. Exp Brain Res 154:97-102.

Guillery RW (2005) Anatomical pathways that link perception and action. Prog Brain Res 149:235-256.

Head H, Holmes G (1912) Sensory disturbances from cerebral lesions. Brain 34:102-254.

Jain N (2002) Adult brain plasticity-what is revealed is exciting, what is hidden is critical. J Biosci 27:439-442.

Jain N, Florence SL, Kaas JH (1995) Limits on plasticity in somatosensory cortex of adult rats: hindlimb cortex is not reactivated after dorsal column section. J Neurophysiol 73:1537-1546.

Jain N, Catania KC, Kaas JH (1997) Deactivation and reactivation of somatosensory cortex after dorsal spinal cord injury. Nature 386:495-498.

Jain N, Catania KC, Kaas JH (1998) A histologically visible representation of the fingers and palm in primate area $3 \mathrm{~b}$ and its immutability following long-term deafferentations. Cereb Cortex 8:227-236.

Jain N, Florence SL, Qi HX, Kaas JH (2000) Growth of new brainstem connections in adult monkeys with massive sensory loss. Proc Natl Acad Sci U S A 97:5546-5550.

Jain N, Qi HX, Collins CE, Kaas JH (2008) Large-scale reorganization in the somatosensory cortex and thalamus after sensory loss in macaque monkeys. J Neurosci 28:11042-11060.

Jones EG (2000) Cortical and subcortical contributions to activitydependent plasticity in primate somatosensory cortex. Annu Rev Neurosci 23:1-37.

Jones EG, Pons TP (1998) Thalamic and brainstem contributions to largescale plasticity of primate somatosensory cortex. Science 282:1121-1125.

Kaas JH (1993) The functional organization of somatosensory cortex in primates. Ann Anat 175:509-518.

Kaas JH (2004) Evolution of somatosensory and motor cortex in primates. Anat Rec A Discov Mol Cell Evol Biol 281:1148-1156. 
Kaas JH, Florence SL, Jain N (1999) Subcortical contributions to massive cortical reorganizations. Neuron 22:657-660.

Kaas JH, Qi HX, Burish MJ, Gharbawie OA, Onifer SM, Massey JM (2008) Cortical and subcortical plasticity in the brains of humans, primates, and rats after damage to sensory afferents in the dorsal columns of the spinal cord. Exp Neurol 209:407-416.

Katz J, Melzack R (1990) Pain "memories" in phantom limbs: review and clinical observations. Pain 43:319-336.

Krubitzer L, Clarey J, Tweedale R, Elston G, Calford M (1995) A redefinition of somatosensory areas in the lateral sulcus of macaque monkeys. J Neurosci 15:3821-3839.

Krupa DJ, Ghazanfar AA, Nicolelis MA (1999) Immediate thalamic sensory plasticity depends on corticothalamic feedback. Proc Natl Acad Sci U S A 96:8200-8205

Lenz FA, Tasker RR, Dostrovsky JO, Kwan HC, Gorecki J, Hirayama T, Murphy JT (1987) Abnormal single-unit activity recorded in the somatosensory thalamus of a quadriplegic patient with central pain. Pain 31:225-236.

Lenz FA, Kwan HC, Martin R, Tasker R, Richardson RT, Dostrovsky JO (1994) Characteristics of somatotopic organization and spontaneous neuronal activity in the region of the thalamic principal sensory nucleus in patients with spinal cord transection. J Neurophysiol 72:1570-1587.

Lenz FA, Garonzik IM, Zirh TA, Dougherty PM (1998) Neuronal activity in the region of the thalamic principal sensory nucleus (ventralis caudalis) in patients with pain following amputations. Neuroscience 86:1065-1081.

Manger PR, Woods TM, Muñoz A, Jones EG (1997) Hand/face border as a limiting boundary in the body representation in monkey somatosensory cortex. J Neurosci 17:6338-6351.

McCandlish CA, Li CX, Waters RS, Howard EM (1996) Digit removal leads to discrepancies between the structural and functional organization of the forepaw barrel subfield in layer IV of rat primary somatosensory cortex. Exp Brain Res 108:417-426.

Melzack R (1971) Phantom limb pain: implications for treatment of pathologic pain. Anesthesiology 35:409-419.

Melzack R (1990) Phantom limbs and the concept of a neuromatrix. Trends Neurosci 13:88-92.

Merzenich MM, Kaas JH, Wall J, Nelson RJ, Sur M, Felleman D (1983a) Topographic reorganization of somatosensory cortical areas $3 \mathrm{~b}$ and 1 in adult monkeys following restricted deafferentation. Neuroscience 8:33-55.

Merzenich MM, Kaas JH, Wall JT, Sur M, Nelson RJ, Felleman DJ (1983b) Progression of change following median nerve section in the cortical representation of the hand in areas $3 \mathrm{~b}$ and 1 in adult owl and squirrel monkeys. Neuroscience 10:639-665.

Merzenich MM, Nelson RJ, Stryker MP, Cynader MS, Schoppmann A, Zook JM (1984) Somatosensory cortical map changes following digit amputation in adult monkeys. J Comp Neurol 224:591-605.

Mishkin M (1979) Analogous neural models for tactual and visual learning. Neuropsychologia 17:139-151.

Murray EA, Coulter JD (1981) Organization of corticospinal neurons in the monkey. J Comp Neurol 195:339-365.

Nelson RJ, Sur M, Felleman DJ, Kaas JH (1980) Representations of the body surface in postcentral parietal cortex of Macaca fascicularis. J Comp Neurol 192:611-643.

Padberg J, Cerkevich C, Engle J, Rajan AT, Recanzone G, Kaas J, Krubitzer L (2009) Thalamocortical connections of parietal somatosensory cortical fields in macaque monkeys are highly divergent and convergent. Cereb Cortex 19:2038-2064.

Parker JL, Dostrovsky JO (1999) Cortical involvement in the induction, but not expression, of thalamic plasticity. J Neurosci 19:8623-8629.

Pettit MJ, Schwark HD (1993) Receptive field reorganization in dorsal column nuclei during temporary denervation. Science 262:2054-2056.

Pons TP, Garraghty PE, Friedman DP, Mishkin M (1987a) Physiological evidence for serial processing in somatosensory cortex. Science 237:417-420.

Pons TP, Wall JT, Garraghty PE, Cusick CG, Kaas JH (1987b) Consistent features of the representation of the hand in area $3 \mathrm{~b}$ of macaque monkeys. Somatosens Res 4:309-331.
Pons TP, Garraghty PE, Mishkin M (1988) Lesion-induced plasticity in the second somatosensory cortex of adult macaques. Proc Natl Acad Sci U S A 85:5279-5281.

Pons TP, Garraghty PE, Ommaya AK, Kaas JH, Taub E, Mishkin M (1991) Massive cortical reorganization after sensory deafferentation in adult macaques. Science 252:1857-1860.

Pons TP, Garraghty PE, Mishkin M (1992) Serial and parallel processing of tactual information in somatosensory cortex of rhesus monkeys. J Neurophysiol 68:518-527.

Ramachandran VS (1993) Behavioral and magnetoencephalographic correlates of plasticity in the adult human brain. Proc Natl Acad Sci U S A 90:10413-10420.

Ramachandran VS, Rogers-Ramachandran D (2000) Phantom limbs and neural plasticity. Arch Neurol 57:317-320.

Ramachandran VS, Stewart M, Rogers-Ramachandran DC (1992) Perceptual correlates of massive cortical reorganization. Neuroreport 3:583-586.

Rasmusson DD, Louw DF, Northgrave SA (1993) The immediate effects of peripheral denervation on inhibitory mechanisms in the somatosensory thalamus. Somatosens Mot Res 10:69-80.

Rausell E, Jones EG (1991) Histochemical and immunocytochemical compartments of the thalamic VPM nucleus in monkeys and their relationship to the representational map. J Neurosci 11:210-225.

Rausell E, Bickford L, Manger PR, Woods TM, Jones EG (1998) Extensive divergence and convergence in the thalamocortical projection to monkey somatosensory cortex. J Neurosci 18:4216-4232.

Reilly KT, Sirigu A (2008) The motor cortex and its role in phantom limb phenomena. Neuroscientist 14:195-202.

Robinson CJ, Burton H (1980) Somatotopographic organization in the second somatosensory area of M. fascicularis. J Comp Neurol 192:43-67.

Schneider RJ (1990) Loss of information concerning hair displacement and other somatic stimuli in the first somatic sensory cortex of unanesthetized Macaca mulatta monkeys following dorsal funiculus transections. Exp Brain Res 83:105-114.

Sengelaub DR, Muja N, Mills AC, Myers WA, Churchill JD, Garraghty PE (1997) Denervation-induced sprouting of intact peripheral afferents into the cuneate nucleus of adult rats. Brain Res 769:256-262.

Stevens RT, London SM, Apkarian AV (1993) Spinothalamocortical projections to the secondary somatosensory cortex (SII) in squirrel monkey. Brain Res 631:241-246.

Sweet WH (1975) "Phantom" sensations following intraspinal injury. Neurochirurgia (Stuttg) 18:139-154.

Tandon S, Kambi N, Jain N (2008) Overlapping representations of the neck and whiskers in the rat motor cortex revealed by mapping at different anaesthetic depths. Eur J Neurosci 27:228-237.

Turnbull BG, Rasmusson DD (1991) Chronic effects of total or partial digit denervation on raccoon somatosensory cortex. Somatosens Mot Res 8:201-213.

Wall JT, Cusick CG (1984) Cutaneous responsiveness in primary somatosensory (S-I) hindpaw cortex before and after partial hindpaw deafferentation in adult rats. J Neurosci 4:1499-1515.

Wall JT, Kaas JH, Sur M, Nelson RJ, Felleman DJ, Merzenich MM (1986) Functional reorganization in somatosensory cortical areas $3 \mathrm{~b}$ and 1 of adult monkeys after median nerve repair: possible relationships to sensory recovery in humans. J Neurosci 6:218-233.

Wong-Riley M (1979) Changes in the visual system of monocularly sutured or enucleated cats demonstrable with cytochrome oxidase histochemistry. Brain Res 171:11-28.

Xu J, Wall JT (1997) Rapid changes in brainstem maps of adult primates after peripheral injury. Brain Res 774:211-215.

Xu J, Wall JT (1999) Evidence for brainstem and supra-brainstem contributions to rapid cortical plasticity in adult monkeys. J Neurosci 19:7578-7590.

Zhang HQ, Murray GM, Turman AB, Mackie PD, Coleman GT, Rowe MJ (1996) Parallel processing in cerebral cortex of the marmoset monkey: effect of reversible SI inactivation on tactile responses in SII. J Neurophysiol 76:3633-3655. 